\title{
Tangence
}

\section{Depuis moi, jusqu'à moi : Dernier accrochage de Diane-Monique Daviau}

\section{Anna Paola Mossetto}

Numéro 50, mars 1996

Lectures de nouvelles québécoises

URI : https://id.erudit.org/iderudit/025894ar

DOI : https://doi.org/10.7202/025894ar

Aller au sommaire du numéro

Éditeur(s)

Tangence

ISSN

0226-9554 (imprimé)

1710-0305 (numérique)

Découvrir la revue

Citer cet article

Mossetto, A. P. (1996). Depuis moi, jusqu'à moi : Dernier accrochage de

Diane-Monique Daviau. Tangence, (50), 85-100. https://doi.org/10.7202/025894ar d'utilisation que vous pouvez consulter en ligne.

https://apropos.erudit.org/fr/usagers/politique-dutilisation/ 


\section{Depuis moi, jusqu'à moi : Dernier accrochage de Diane-Monique Daviau}

\section{Anna Paola Mossetto}

Dans le vivant tableau des nouvelliers québécois contemporains, Diane-Monique Daviau occupe une place de premier plan, quant à la finesse des instruments et à la cohérence de l'imaginaire qui est chez elle foisonnant et nuancé.

Le (la) nouvellier(ère) est celui (celle) qui « est pleinement engagé(e) dans une démarche nouvellière "1, nous apprend en connaissance de cause André Carpentier. Il en est ainsi pour cette auteure dont l'œuvre révèle un talent nettement orienté vers l'expérimentation de l'écriture de la brièveté, de la rupture et de la reprise. Diane-Monique Daviau privilégie depuis une quinzaine d'années la pratique nouvellière tant par sa collaboration à des collectifs ou à des revues spécialisées que par le regroupement de ses textes en recueils.

Un aperçu d'ensemble de ce corpus m'amène à rappeler, en faisant toujours référence à l'étude de Carpentier sur les genres narratifs brefs, que les deux aspects fondamentaux de l'écriture nouvellière sont "le caractère fragmentaire" et "le principe de discontinuité par assemblage de brièvetés" ${ }^{2}$. C'est à partir de ces observations générales, désormais bien acquises, ainsi que des remarques souvent citées de Paul Zumthor sur "l'aspiration au regroupement " ${ }^{3}$ des fictions brèves, ou encore des réflexions de Jean-Pierre Boucher sur le statut "grégaire" de la nouvelle ${ }^{4}$, que je vais faire porter mon attention sur une série textuelle (le

1 André Carpentier, - Commencer et finir souvent. Rupture fragmentaire et brièveté discontinue dans l'écriture nouvellière -, La nouvelle: Écriture(s) et lecture(s), Montréal, XYZ éditeur, 1993, p. 35. Pour des raisons d'euphonie et de cohérence, je partage la prédilection d'André Carpentier pour le terme * nouvellier * (qui est en lien de suffixe avec romancier), par rapport à celui de "nouvelliste (qui rime avec journaliste).

2 Ibid., p. 36.

3 Paul Zumthor, Essai de poétique médiévale, Paris, Seuil, 1972, p. 404.

4 Jean-Pierre Boucher, Le recueil de nouvelles: études sur un genre littéraire dit mineur, Montréal, Fides, 1992, p. 10. 
86

recueil) plutôt que sur un texte singulier. Je m'engage donc dans une quête du sens (ou mieux des sens) émergeant du recueil Dernier accrochage de Diane-Monique Daviau, attirée par la dynamique du processus de signification qui ne manque de s'instaurer entre "genre bref ", générateur d'hétérogène, et "genre long", à fonction cotextualisante ${ }^{5}$.

Ce regroupement de nouvelles, qui a paru en tant que livre en 1990 aux éditions XYZ, dans la collection "L'Ėre nouvelle" 6 , se compose de seize nouvelles, dont douze ont fait l'objet d'une publication antérieure, à partir de 1986, dans des périodiques ou des rassemblements allographes. C'est le cas aussi de la nouvelle éponyme, éditée quatre ans auparavant dans le collectif monothématique Aimer?.

Il est bien évident que le recours à la taxinomie n'a d'importance qu'en fonction du discours sur la production de sens par le texte: c'est dans ce but que je vais poser des étiquettes. Le recueil de Diane-Monique Daviau, cependant, s'impose plus par ses divergences qu'en raison de son appartenance à des catégories. Bien que fruit d'un projet postérieur à la rédaction de la totalité des nouvelles, il se situerait entre le "recueil thématique" et le "recueilensemble", selon la typologie élaborée par Jean-Pierre Boucher ${ }^{8}$. Quant à l'affinité des sujets, la quatrième de couverture aborde la question en faisant mine de la justifier, mais en réalité, elle laisse le lecteur à l'entrée d'un labyrinthe, l'invitant à creuser toutes sortes de pistes suggérées par le jeu croisé des homonymes, des paronymes, des synonymes déclenchés par des variations sur le titre. En fait, ce recueil étale son unité structurale et non seulement thématique car, malgré les coordonnées très succinctes offertes par les éléments du paratexte sur les récits prépubliés en ordre épars, on peut en déduire que leur reprise dans le recueil n'a pas été faite selon l'ordre chronologique; leur redistribution dans les trois divisions intérieures obéit plutôt à un dessein englobant, expressément mis en œuvre pour ce recueil de façon à construire un tout organique.

La table des matières, en outre, montre que les trois sections rassemblent chacune un nombre différent de nouvelles: sept

5 Voir André Carpentier, op. cit., p. 38

6 Les références aux nouvelles seront tirées de cette édition et désormais indiquées entre parenthèses.

7 Collectif dirigé par André Carpentier, Montréal, éditions Quinze, 1986.

8 Voir Jean-Pierre Boucher, op. cit., p. 15. 
dans la première, cinq dans la seconde, quatre dans la troisième. II s'agit, néanmoins, d'une disparité rééquilibrée par la longueur des textes inscrits dans chaque partie, ce qui vient rétablir une certaine symétrie des proportions: on peut compter ainsi une soixantaine de pages pour chacun des volets.

Ces dimensions quantitatives du recueil ne me paraissent pas dépourvues d'intérêt dans la mesure où elles dénotent une forte architecture et, en même temps, l'intention de l'auteure de subdiviser son discours narratif par trois indicateurs de zones. Cela n'empêche pas la fluidité de lecture, mais pose tout autant l'avertissement du passage à d'autres territoires. Le vieux poncif de la lecture comme errance est ici prêt à resurgir, secondé par la carte de cet archipel, où les titres énoncent le schéma d'une ligature assez précise de ces fictions brèves, qui ne cessent pour cela d'exister en tant que "cheminements multiples et diversifiés " 9 .

Toujours est-il que l'espacement infratextuel et la mise en relief typographique des intertitres, à la fois dans la table des matières et dans la composition du livre, produisent des entités définies à l'intérieur du recueil, dont la disproportion serait génératrice de sens. Si l'on compare les repères bibliographiques (p. 170) à la table des matières (p. 171), on note que les nouvelles déjà parues en revue sont au nombre de quatre dans chacune des trois sections, avec trois ajouts d'inédits dans la première et un dans la seconde. Ces additions, qui introduisent une disparité dans la chronologie de parution, apportent par contre un aménagement mieux balancé dans les trois subdivisions.

Il n'y a donc aucune mise en relief de l'une en particulier des trois parties, "Solitudes", Alliances", - Liens", qui se posent et se composent en relation dialectique et en tension continuelle. Elles ouvrent trois éventails paradigmatiques par rapport au titre général du recueil, où l'effet de séquence n'entraîne pas nécessairement celui de progression et où les confrontations sont possibles dans toutes les directions. La présentation matérielle du recueil prélude à la circularité de la signification qui sera mise en évidence à travers l'examen des récits.

Pour filer la métaphore de la lecture-itinéraire, remarquons encore que le titre général nous propose la synthèse d'une

9 André Carpentier, op.ctt., p. 40. 
88

indication de temps et d'une indication de lieu, deux coordonnées essentielles à toute mouvance, à tout voyage. Avançons donc en dégageant la route d'un autre leurre.

Le titre du recueil n'est qu'apparemment emprunté à celui d'une nouvelle, "Le dernier accrochage", car la suppression de l'article modifie quelque peu le sens du syntagme en produisant le plein déploiement de sa polyvalence sémantique. De plus, la nouvelle éponyme (ou presque), elle aussi tient de l'inusité par la position anormale où elle est confinée: en tant qu'avant-dernière, elle perturbe les combinaisons les plus traditionnelles qui la voudraient en ouverture ou en clôture du recueil. Normalement, l'analogie avec le titre du recueil la démarquerait des autres, tandis que la mutilation partielle de son propre titre dans la désignation du recueil la replongerait dans l'uniformité. Son hégémonie est signalée et en même temps mise en question par l'option du titre général qui l'évoque tout en la modifiant, et par son lieu d'insertion dans l'organisation de l'ensemble, qui est néanmoins lourd de sens. Sa place d'avant-dernière nouvelle empêche, en effet, tout climax libératoire, reproduisant ainsi l'instabilité du doute systématique, et prenant forme de parabole de la perpétuelle remise en question. Nettement plus longue que les autres, elle introduit un élément de déséquilibre de ce point de vue aussi.

On pourrait en déduire que la construction bancale du recueil, qui est assurément intentionnelle, compte tenu par ailleurs de l'attention portée par l'auteure aux rapports de symétrie et de correspondance, reflète et renforce les caractéristiques de l'écriture nouvellière, puisque la nouvelle rend compte, selon le mot de Jean-Pierre Boucher, "des limites, voire de l'impossibilité ou du refus d'une vision du monde unifiée synthétique. Éclatement, relativité, mouvement, questionnement, voilà quelques-uns des aspects de la sensibilité contemporaine que permet d'exprimer le recueil " ${ }^{10}$. Cependant, si André Carpentier, dans un récent bilan, prend appui sur cette interprétation en écrivant que "le choix de l'écriture nouvellière est justement affaire de lecture du monde "11, ce qu'il ajoute quelques paragraphes plus loin, dans le même essai, me semble être encore plus révélateur: "L'écriture nouvellière, affirme-t-il, c'est l'écriture en refonte spasmée d'identité. "12

10 Jean-Pierre Boucher, op. cit., p. 40.

11 André Carpentier, op. cit., p. 36.

12 Ibial, p. 44. 
Dans Dermier accrochage, il ne faut pas prioritairement chercher les pièces d'une enquête sur la réalité humaine grouillante de gens en manque de liaisons ("Solitudes"), ou complices plus ou moins éphémères ("Alliances"), ou encore en état de dépendance affective (*Liens "), mais il convient d'y mettre en relief un réseau de cheminements qui convergent vers une prise de conscience de soi toujours problématique.

Très révélatrice apparaît, d'autre part, l'indication offerte par Diane-Monique Daviau elle-même, lorsqu'en répondant à l'enquête sur la nouvelle menée par Québec français en mai 1987 auprès d'un certain nombre de nouvelliers, elle explicite ainsi la raison de son choix:

Mon faible pour la nouvelle vient du fait que ce genre littéraire permet mieux que d'autres la confrontation. [...] La nouvelle appelle à se confronter soi-même avec chaque phrase, chaque image, chaque mot, parce qu'elle repose justement sur la confrontation serrée de chaque phrase avec la précédente et la suivante. Je crois que ce travail des différents éléments entre eux n'est réussi ou du moins satisfaisant que si l'on accepte, tout au long de la rédaction d'une nouvelle, de se colleter soi-même sans ménagement avec chaque élément qui compose cette nouvelle. ${ }^{13}$

De cet éclaircissement, j'aimerais tirer un fil d'Ariane, le sentiment du moi, qui me permettra d'entrer dans le palais mythique (toute pratique narrative a une dette à cet égard), d'en affronter le monstre (au sens étymologique de "merveille ", bien sûr) et peutêtre d'en sortir indemne.

Dans Dernier accrochage, toutes sortes d'accrocs, de crochets, de chances, d'aides, de déchirures, d'accidents, etc., qui semble mettre soudainement l'individu en rapport avec la réalité extérieure ne sont que la manifestation d'une quête ayant le déchiffrement du moi comme seul et unique objet. Et cela, non seulement au niveau des aventures existentielles où agissent les personnages, mais surtout sur le plan autrement significatif des instances narratives.

* Chacun vit, sent et pense selon une temporalité, une spatialité, une relationalité qui lui est particulièren, écrit Georges Poulet en étudiant les composantes de la :grande activité catégorielle de

13 - Les nouvellistes réfléchissent sur la nouvelle., Québec français, n 66 , mai 1987, p. 60. 
90

la conscience de soi." ${ }^{14}$ Mais d'après les essais où il s'attache à relever les variations de l'acte de se penser chez les écrivains français du $\mathrm{XVHII}^{\mathrm{e}}$ au $\mathrm{xx}^{\mathrm{e}}$ siècle, il ressort que la conscience de soi peut apparaître principalement sous deux aspects qui tendent à s'opposer. Il y a, d'un côté, une saisie purement intellectuelle de soi-même, qu'on peut pousser jusqu'au fait d'une activité spirituelle dépersonnalisée, et il y a, d'un autre côté :

moi qui me sens, qui me saisis moi-même plus ou moins confusément dans le sentiment primitif que j'ai de mon existence, j'aperçois de moi une image incertaine mais le plus souvent attirante, qui se confond volontiers avec des états d'âme troubles, des émotions vives mais transitoires, tout le jeu de l'expérience sensible qui est la mienne. ${ }^{15}$

Cette dernière connaissance intime, grâce à laquelle je me découvre existant, tient d'un héritage romantique qui prend souvent, dans les temps modernes, les accents de l'angoisse et de la peur face aux limites des facultés cognitives humaines. Dans le recueil de Diane-Monique Daviau, deux nouvelles surtout nous offrent des exemples très évidents de cette forme affective d'appréhension de son moi.

"Les inséparables" est un monologue aux tons exaltés, exposant les sensations, les obsessions, les hallucinations d'un personnage anonyme qui, le jour, maintient son équilibre instable grâce à des données réelles: "En pleine lumière je me raisonne. Je sais distinguer le réel de tout ce qui est flou. Je sais qui je suis, où je vis. Je sais que là où je vis coule une rivière. (p. 34). Tandis que la nuit, ses cauchemars l'assaillent sous la forme effrayante de deux "somnivores". Il essaie de se recomposer une identité de plus en plus schizophrénique, jusqu'à désirer être complètement aliéné par ses envahisseurs de l'intérieur: *[I]ls sont [...] davantage moi que je ne le suis moi-même. Moi, il me reste seulement la surface, les apparences. (p. 36). Notons que la narration progresse en parallèle avec la diégèse: c'est une fabulation solitaire où la première personne est continuellement repoussée par l'ingérence de la troisième personne et de formes verbales impersonnelles: * Ils reviennent tous les soirs. [...] Ça crie en eux, on l'entend de loin. (p. 33).

14 Georges Poulet, Entre moi et moi: essai critique sur la conscience de soi, Paris, José Corti, 1977 , p. 274.

15 Ibid., p. 275. 
Une pareille crise d'identité, une crise moins pathologique mais faisant l'objet d'une confession tout aussi insolite, et peutêtre encore plus inquiétante puisque plus proche du seuil de la norme et du quotidien, est le sujet de la nouvelle "Tout est doux.. C'est le récit à la troisième personne d'une attaque de mal du pays qu'un Montréalais non identifié subit soudain au milieu de ses vacances autrichiennes: *Tout à coup, quelque chose brisa la perfection du paysage, de la vie, de l'amour qui l'avait entraîné là, quelque chose de minuscule annula l'harmonie de sa petite vie et le contentement qu'il en avait tiré pendant plusieurs saisons" (p. 53). Mais rentré, puis de nouveau reparti, il se rend compte qu'au fil de sa vie il ressent la même nostalgie pour tous les ailleurs qu'il a fréquentés: "Il erra longtemps ainsi, partagé, déchiré. Se retrouva, de pays en pays, à l'autre bout du monde. Fatalement étranger. Prisonnier de sa peine. (p. 58). Tout le champ de l'expérience est occupé par une somme de sensations intenses, lesquelles, au lieu de fournir au sujet une connaissance du monde, déclenchent une hypertrophie de la faculté introspective. C'est l'empire du sentiment de soi, capable d'annuler le poids de la rencontre avec des "gens passionnants" ou des "lieux bouleversants. L'espace est donc le lieu de l'interrogation du moi, mais aussi celui de sa dispersion.

La faiblesse ontologique se révèle également dans la dimension temporelle des êtres: la poursuite d'un point de repère se résout une fois de plus par la prise de conscience de l'écartèlement de soi. Le "je " des "Inséparables" déborde et se détraque dans l'obsession d'un "désastre quotidien. à fuir par les voies de "vies précédentes" et de "vies à venir"; le Montréalais de "Tout est doux", baigne dans un bien-être raconté d'abord à l'imparfait, puis au présent figé et éternel: «[II] n'arrive qu'à souffrir. Partout, tout le temps. Hier, aujourd'hui, le bonheur ne dure pasn (p. 58).

Dans "Le psychiatre jouera du piano", une telle notion phénoménologique du temps déstabilise la conscience du moi au lieu de la déterminer. Il est toujours question de temps implosif dans cette interview accordée par une femme médecin du service d'urgence à propos du cas d'un de ses collègues qui aurait poussé un homme en proie au stress à se suicider: *Le temps de l'urgence, surtout en psychiatrie, est un temps suspendu" (p. 100); la dépression est un atemps perdu à vivre à côté de ses souliers" (p. 107). 
92

Bien que sur un registre narratif un peu différent, utilisant davantage les tons de lhumour et du grotesque que ceux du pathos et du drame, la nouvelle "Tante Lili ou le caviar des petits lapins" raconte aussi une recherche de soi fondée sur la perception subjective du temps, et finalement trahie par elle. C'est l'histoire, abrégée dans les souvenirs de sa nièce, d'une vieille fille "convaincue d'avoir vu le jour sous une mauvaise étoile" (p. 42), mais croyant en la vertu miraculeuse des trèfles à quatre feuilles qu'elle sème patiemment, et longtemps sans résultats, dans l'espoir de faire tourner la chance du bon coté. Mais "un mardi en fin d'après-midi", lorsqu'elle vient d'en trouver une poignée, elle meurt d'un banal accident. C'est alors que la nièce prend la relève de sa tante pour compléter les connaissances de celle-ci en matière de bon et de mauvais sort, connaissances trop insuffisantes et donc inefficaces à soutenir une existence si éphémère. Pendant ses visites, la fillette pense, en effet, que l'aspect le plus réel de sa tante est le bruit de ses pendants d'oreilles: "Il me prouvait qu'elle existait, que, malgré tous ses malheurs, elle n'était pas encore un fantôme. (p. 42). Mais la nièce va apprendre que c'est justement ce seul élément concrètement identitaire de Tante Lili qui l'a perdue, elle qui s'était trouvée en opposition dérisoire avec la dimension temporelle, car "on ne doit jamais, au grand jamais, porter de lourds bijoux un mardi de pleine lune (p. 46).

Une contre-épreuve du désaccord entre les coordonnées spatio-temporelles et tout élan vers la récupération de sa propre unité intérieure est implicite dans "Hôtel des chats de mer". Une créature malade d'ennui, vagabonde, insomniaque, anorexique, apathique se régénère en branchant son ego à des illusions produites par l'invention mi-thérapeutique mi-amicale d'un médecin humaniste puisant à toutes les ressources de la technologie. Cette espèce d'ange gardien (l'hôtel où elle trouvera son salut se situe bien sur le boulevard Saint-Gabriel) organise pour elle, à son insu, une scénographie complète de figurants, et programme son emploi du temps à l'aide d'une bande enregistrée: "Axel avait prêté sa voix grave et chaleureuse à cette petite pendule ornée d'un chaton qui bâille et avait ainsi réglé le problème angoissant de mes retours quotidiens dans le monde des réalités quotidiennes " (p. 74).

Le moi s'approprie ce système chronotopologique mécanique comme une prothèse devant assurer la remise en état d'une psyché sur le bord de sombrer: "J'aurais sûrement lâché prise" (p. 67). La narration est solidaire de l'histoire: l'artifice des procé- 
dés linguistiques, toujours sous-jacents dans l'écriture de DianeMonique Daviau, est ici plus apparent; il joue sur les motifs du "chat " et de "l'eau "juxtaposés dans le titre (depuis la comparaison de l'hôtel-château - chat d'eau - parcouru d'un "dédale de couloirs, véritable écheveau de fil brouillé par un chat", jusqu'à des locutions comme "sommeil de chat").

Si, parmi les signes mis en action dans ce recueil, les données sensibles de la réalité environnante et leur reproduction artificielle ont une fonction de support de la représentation intellectuelle que les êtres humains se font d'eux-mêmes, le mode catégoriel de la relation avec les autres, lui, semble, dans l'esprit humain, le mieux permettre de vérifier la résurgence de l'idée du moi dans l'individu. Cependant, sur ce point encore, Dernier accrochage dépasse la commune mesure. D'une part, certaines nouvelles aboutissent à l'attestation de l'impossibilité pour les hommes de nouer avec les autres des liaisons vraiment respectueuses de l'authenticité et de l'indépendance réciproques; d'autre part, le moi du narrateur tend toujours à prévaloir. C'est ce qui arrive, dans "Pas mon bébé", où une mère de famille, qui jouit de maintes satisfactions professionnelles, sociales et familiales, devant le désir de son troisième enfant de devenir "écrivain et rien d'autre", se souvient d'un épisode de sa jeunesse. Pendant une période difficile de ses études en orthodontie, elle avait eu comme voisin d'appartement un romancier "pauvre et démuni", avec qui elle s'était momentanément liée d'amitié: "l'anxiété, le désarroi me rendaient vulnérable, réceptive, plus que compréhensive: empathique. J'étais capable de me mettre à la place de cet homme. Cet homme, je savais exactement ce qu'il ressentait "(p. 30). Mais ensuite, il y a eu séparation et le succès a récompensé le choix d'une vie mieux réglée selon des projets égocentriques: "Puis tout rentra peu à peu dans l'ordre: je passai mon examen et la réussite effaça rapidement ces mauvais souvenirs. (p. 31). L'attention pour les autres n'est qu'un faux altruisme, car le moi tire prétexte de toute liaison soit pour s'approprier, soit pour prévaloir. "Ce n'est vraiment pas ce que j'avais rêvé pour elle. Je ne peux pas croire que ma propre fille va vivre çă" (p. 32). Ce récit à la première personne est riche en humour savamment créé par l'emploi d'un langage désinvólte qui dénote une forte maîtrise de la réalité : l'autonomie et l'hégémonie du moi en est la source.

"Comprendre" met en scène un je narrant, marginal par comparaison aux personnages principaux de l'action, mais qui veut 
94

dompter la situation grâce à une conduite sévère, dictée par la mésentente des rapports entre enfants et personnes âgées. C'est encore un ego qui prétend s'affirmer en se substituant à quelqu'un d'autre, sans être capable en réalité de se mettre à la place d'autrui.

Il en est de même dans "Roses théières.", où une psychiatre raconte comment une de ses jeunes patientes, victime d'une tragédie familiale, est en train de recomposer son moi broyé et de retrouver son équilibre psychique en suivant un chemin tout à fait original et indépendant de la thérapie. Cette rencontre entre infirme et médecin amène surtout celle-ci à mettre en accusation sa "formidable incompréhension", malgré l'utilisation d'une "techniqué parfaite". Dans cette franche déclaration de faillite, c'est encore le moi de la psychiatre qui fait l'objet d'une enquête et donc d'une mise en relief. Le moi de la petite se pose en tant qu'interlocuteur, mais sans un vrai dialogue: il renaît indépendamment, d'abord, comme il se doit, par une identification physique à la mère (qu'elle a délivrée en supprimant le père sadique) : "Le père avait les cheveux blonds, elle tue le père, elle perd ses cheveux. Lorsqu'ils repoussent, ils deviennent peu à peu noirs comme ceux de la mère. Pour qui elle a tué le père à bout portant " (p. 113). La rencontre avec les autres ne se produit donc pas dans les termes d'un échange enrichissant; elle se présente plutôt comme un miroir narcissique, voire comme une appropriation du monde, nourricière de l'image de soi en train de se définir.

La structure narrative de "Brisa Siblewskin est exemplaire à ce propos. Par une énonciation à la troisième personne, cette nouvelle peint le portrait d'une femme bizarre, dont le nom est affiché dans le titre, qui *se faufile tous les jours dans la salle d'audience où sont présentées les demandes de divorce" (p. 49), dans l'espoir d'y rencontrer le "candidat idéal " pour un mariage réussi. Brisa s'autodéfinit comme une "FEMME " en majuscules, mais son ego confirmé obtient comme résultat la révélation d'un autre moi, celui qui se cache derrière l'instance narrative et qui, grâce à cette influence, se manifeste tout à coup : * Et moi je suis cette histoire de très près, car Brisa me fascine" (p. 48).

Encore plus dévorantes sont les liaisons qui s'établissent entre le personnage principal de "L'œil de Lily" et son entourage, ou entre la protagoniste des "Poupées d'Émilie" et le docteur Rémillard, qui soigne Émilie au département de psychiatrie infantile. Dans le premier cas, un photographe hanté par le souvenir des 
regards sévères que ses parents ont toujours jetés sur ses faiblesses d'enfant, provoquant chez lui un blocage dans la formation fondamentale de son sentiment d'auto-estime, cherche des échappatoires au malaise qu'il éprouve pendant une séance de psychanalyse, en fixant ses yeux sur différents objets dans la tentative péniblement chaotique de recomposer son identité émiettée. Mais la réalité extérieure ne lui permet d'accrocher sa propre image intime qu'à l'oil "exagérément maquillé, lourd et vide" de Lily, le mannequin qu'il mitraille avec son appareil de photos, tout en s'y reconnaissant en un mouvement d'autocommisération: "Pauvre Lily, dit-il, pauvres petites Lily de mon coeur!n (p. 86) Les deux syllabes de ce prénom apparaissent comme l'icône du visage de la fille, maquillé seulement à moitié pour les besoins de la publicité, et aussi comme la représentation inachevée et factice du moi de l'homme.

Toujours parmi les variations de l'extension du moi sur autrui, il y a le cas d'Émilie Brouillard, dont l'ego s'impose, par contre, comme le fruit de la rencontre réussie entre ses troubles d'enfant schizophrène et l'assurance naïve du médecin qui la soigne. Émilie rédige un rapport pour le directeur de l'hôpital "sur le traitement que nous venons de terminer, moi et le docteur Rémillard " (p. 87), dit-elle, et dont la réussite tient à un double transfert avec dominance de la composante mythomane. Les deux moi se croisent et se substituent : l'homophonie des noms est un indice.

Question de changement de rôles, aussi, dans "Vous", récitdialogue tissé serré entre les membres d'un vieux couple plutôt solide, mais dont la femme décide de passer au vouvoiement pour rafraîchir une vie en commun vieille de vingt-cinq ans. Elle sappelle Constance et ressent un besoin de changement vital. Lui s'appelle Vital et fait preuve d'une plus grande constance. Bien qu'insignifiants, ce sont des personnages à vocation totalisante, du moins sur le plan de la production de sens liée aux patronymes. Mais qui avale qui?

La solidarité envers les autres est une fois de plus un prétexte à une confession personnelle, dans "Le psychiatre jouera du pianon: la personne interrogée finit par parler très peu du psychiatre sur lequel porte l'enquête, privilégiant ses propres impressions, ses émotions, son agressivité. Ce dossier se constitue finalement comme un acte d'attestation du moi de la locutrice.

Mais une personnalité encore plus exclusive est celle du protagoniste de "L'imprévisible", qui croit pouvoir gérer l'existence 
96

de sa femme jusqu'au-delà de sa propre mort: il éclate littéralement en apprenant qu'au contraire, c'est elle qui l'avait remplacé et contourné durant toute leur vie.

Dans ce bilan trop rapide, qui m'a permis de tracer quelques lignes de force sur la question de l'exercice de l'acte de conscience à travers les parcours de Dernier accrochage, j'ai expressément laissé de côté trois nouvelles, la première, la dernière et l'éponyme, que je vais commenter maintenant comme les piliers de la structure d'ensemble, dont elles synthétisent les éléments fonctionnels en les portant à leur plénitude sémantique.

"Tout est question de lieu. Ou de temps?" (p. 137), sert d'incipit à la nouvelle "Le dernier accrochage. Deux des principales composantes morphologiques des histoires racontées, indices essentiels de toute représentation intellectuelle, sont ici évoquées, confrontées et mises en discussion. J'ai déjà souligné que l'espace référentiel, dans ce recueil, ne sert que peu souvent de décor aux nombreux développements descriptifs, et que les lieux ne revêtent que rarement une configuration exprimée avec précision. Plus importantes apparaissent ses qualités d'espace fonctionnel et signifiant ${ }^{16}$. Quant à la durée chronologique des anecdotes, elle est soumise à la norme ainsi résumée par Daniel Grojnowski: * Comme le temps du théâtre ou du cinéma de fiction, le temps de la nouvelle se définit par son unité dramatique. "17

Les auteurs peuvent cependant mettre en œuvre des durées concentrées, dilatées ou brouillées. Très peu nombreuses sont les nouvelles de Diane-Monique Daviau qui condensent la durée d'une existence en un récit de vie; elle sont le plus souvent des exemples, pour reprendre l'expression de Michel Lord, "d'un degré zéro de problématisation de l'événementiel " ${ }^{18}$, c'est-à-dire construites autour d'un seul épisode. Tel se présente "Le dernier accrochage ", tandis que la première et la dernière nouvelle s'apparentent davantage au récit-bilan.

La nouvelle éponyme met en scène le croisement des destins de quelques participants à un souper rituel après une conférencevernissage, le dernier accrochage de tableaux, événement vu par les yeux de l'un des personnages. Celle qui perçoit et narre l'ac-

16 Voir Daniel Grojnowski, Lire la nouvelle, Paris, Dunod, 1993, p. 79.

17 Ibid., p. 88.

18 Michel Lord, a Fragments d'univers ", Québec français, n 66, mai 1987, p. 30. 
tion montre un sentiment aigu de l'espace, perçu comme un endroit chaotique et trompeur: le repas se déroule autour de trois tables dans la "salle aux miroirs" du restaurant. De même, le temps n'offre aucun jalon concret. On y lit: a je venais de faire un pas de plus vers la paresse du hasard qui n'existe pas et qui allait pourtant me mener, doucement, au cour de l'impossible " (p. 140); et encore: “Chaque année on sent mieux que ce n'est pas le temps qui passe de cette manière, mais la vie. (p. 147).

Les relations entre les gens se déploient de manière encore plus irréelle. C'est une histoire de fins d'amours - au pluriel. Une femme est contrainte de se taire pour garder son amant, qui néanmoins va bientôt s'éloigner d'elle; une autre femme, la narratrice, qui ressent l'immobilité et le silence comme une protection pour sa liaison amoureuse, quittera son ami qui lui reproche son repliement sur elle-même. Dans cette nouvelle, comme dans le recueil entier, la parole est paradoxalement synonyme de solitude, d'exclusion des autres, d'égotisme défensif: l'intériorisation du point d'observation prévaut, mais l'affabulation manifeste une grande nostalgie de silence isolant et rassurant. Sur ce dernier seulement semble pouvoir se fonder une quelconque certitude existentielle.

Les mots et leur suspension sont comme "les amoureux du train", récit dans le récit qui pourrait se lire comme une sorte de mise en abyme, insérée dans cette nouvelle, du procès d'écriture nouvellière et de son implicite recherche perpétuelle entre parole et silence. Afin de renouveler leur rapport passionnel, raconte Iso, deux amoureux décident de jouer ainsi: ayant acheté une carte valable pour tout le réseau du pays, ils partent chacun de leur côté et sans connaître le trajet du partenaire. Il faut "qu'ils tombent sur la même ville pour avoir le droit de se retrouver et de poursuivre le voyage ensemblen (p. 149). Ce qui est angoissant et stimulant, comme tout véritable rapport amoureux.

S'éloigner pour se retrouver est également la philosophie mise en pratique par le couple de "Vous": «Elle avait remarqué, aussi, qu'en se vouvoyant, les gens prononçaient plus souvent le nom de l'autre, pour contrebalancer la distance respectueuse comprise dans le "vous", pour se rapprocher de l'autre" (p. 132).

La première et la dernière nouvelle, bien qu'exemplaires d'une option énonciative où le témoin s'estompe au profit du témoignage, précisent et complètent ces remarques en les projetant sur l'architecture d'ensemble. On peut les lire comme des 
doubles stratégiquement postés aux deux extrémités du recueil, et leur rapport spéculaire est censé synthétiser un parcours quelque peu évolutif.

"Au logis du Cheval d'Or" relate l'histoire de Marie-Babette, rendue orpheline par un incendie et accueillie par une cousine aubergiste. Mais au moment de descendre du mulet qui l'a menée à sa nouvelle maison, elle "s'écroule sur le sol en poussant un cri affreux * (p. 13). C'est la seule émission de voix qu'on lui connaît, et elle la produit chaque fois qu'on essaie de lui écarter les jambes. À cause de ce traumatisme, elle tuera son époux et sa belle-mère et se jettera par la fenêtre après avoir accouché d'une fille, MarieElisabeth. Celle-ci reprendra ce hurlement lorsque la famille de clowns qui l'a adoptée tentera de l'entrainer comme écuyère. Cette nouvelle inaugurale a l'allure d'un hommage rendu à la tradition des récits brefs du Xxx siècle, surtout quant à l'effet de vraisemblance lié aux indications de dates et de lieux. L'accent, toutefois, n'est pas tellement à mettre sur l'anecdote au parfum fantastique, mais sur ce moi central qui s'énonce d'entrée de jeu dans le recueil par un cri. Cela semble presque la genèse d'une entité personnelle à l'éveil de sa conscience, mais qui ne veut vraiment pas s'expliquer, ni établir des contacts. C'est juste une pulsion qui l'amène à s'affirmer, sans sortir de son opacité jusqu'à la suppression de tout témoin, avant de s'anéantir après s'être multipliée quasiment par une parthénogenèse. Obscure et conflictuelle résurgence d'un ego impérissable. Entre ce moi et le monde, pas de transfert possible, puisque les autres n'offrent qu'un miroir déformant peuplé d'ombres à l'identité imprécise et neutre: "On l'encourage, on rit, on applaudit. [...] On en fera une grande écuyère.[...] On se dit que sa peur disparaitra.[...] Ce n'est qu'une question d'habitude, n'est-ce pas? Une question de temps..." (p. 23)

De même, "Une femme s'en va", dernière nouvelle du recueil, résume l'essentiel d'une vie. La protagoniste est introduite par le titre et par l'incipit comme une figure anonyme et anodine de passage dans un de ces non-lieux de la surmodernité: l'aéroport de Munich, "grand ouvert sur le monde (p. 157), c'est-à-dire sur le vide car, "comme les lieux anthropologiques créent du social organique, les non-lieux créent de la contractualité solitaire "19. Mais quel moi traverse cet espace? Assez léthargique,

19 Marc Augé, Non-lieux, Paris, Seuil, 1992, p. 119. 
habitué au silence, le personnage sursaute lorsqu'on résume son identité " en lui remettant son passeport: Michèle Trock. Quarante ans. Née à Montréal, Québec, au milieu du siècle" (p. 157). Une vie en quelques lignes, détaillée ensuite sur le mode du retour en arrière: un mari, deux enfants qu'elle abandonne une nuit de février 1976, irrésistiblement poussée par un sentiment d'étrangeté, quinze ans vécus dans une ville indifférente, parmi des gens dont elle ne partage pas la langue, guidée par "le vague souhait de sortir du temps et d'entrer dans l'oubli total" (p. 168). Puis c'est l'hospitalité offerte à une jeune ivrogne dans sa chambre en location et l'incendie qui s'ensuit et qui lui fait perdre la main droite: "Alors Michèle Trock s'accroche. Pour ne pas retomber dans son rêve, elle s'accroche à sa vie et oblige ceux qui l'entourent à en faire autant . (p. 167). Cette fois-ci, la connaissance du moi entraîne une épiphanie de la vie universelle qui n'est pas pour autant à considérer comme le résultat peu croyable d'un optimisme superficiel, mais comme le signe d'un progrès plus délié, qui ne perd pourtant pas son caractère aléatoire.

Parmi les récurrences établissant dans ce recueil une symétrie ostensible entre les textes du début et de la fin, il faut signaler l'inversion tant de la suite diégétique que de la perspective de la narration. Au niveau de l'action, dans la première nouvelle, l'incendie représente le drame catastrophique de départ, transformant l'histoire en une destinée gouvernée par l'inéluctable, et à cela contribue la linéarité de la composition chronologique, où la troisième personne est observée par un narrateur extradiégêtique qui emprunte la voie de l'exposé factuel, de telle façon que la netteté de l'exposition se trouve associée à l'énigme du personnage et de son aventure existentielle.

Dans la dernière nouvelle, la péripétie de l'incendie, bien que constituant une expérience violente et douloureuse, provoque dans l'anecdote une virevolte finale positive. 'C'est une modification de direction qui entraîne une narration en flash-back et par conséquent un changement de point de vue, se rattachant davantage aux états de conscience malgré l'énonciation à la troisième personne qui, cependant, se poursuit jusqu'à la conclusion. Et c'est là qu'advient le plus grand bouleversement entre les éléments des deux récits. La manifestation du moi qui, dans le personnage, prend forme d'abord en se situant en creux dans la fuite de sa propre voix "qui sonne faux" (comme il est dit à plusieurs reprises), et se poursuit péniblement dans le balbutiement des 
mots étrangers essentiels, tels que "Z-I-M-M-E-R*, représente une progression par rapport aux manifestations vocales puissantes mais inarticulées qui jalonnent le premier récit. Mais la clausule est soudainement révélatrice d'une étape encore plus avancée où la fin n'est que l'essor pour la reprise. Finir et recommencer "l'illimité du monde" 20 : et voilà que l'enseigne du Logis du Cheval d'Or, qui avait été décrochée par Babette dans sa chute mortelle, se métamorphose en l'affiche qu'" on nettoie, [...] repeint et [...] accroche" (p. 169) dans la chambre à louer de Munich. En outre, la conscience de soi semble moins atteinte par l'imperméabilité de la réalité extérieure : l'espoir en la possibilité d'un accord s'insinue entre la singularité du moi et l'univers environnant. Telle est, au moins, l'image que semble renvoyer le monde, presque un témoignage de sympathie, même si c'est sous la forme d'une hypothèse subrepticement issue de l'instance d'énonciation: "Dans l'avion, au dessus de la rue Schack, la femme se penche et regarde par le hublot. De loin on dirait que la femme sourit. Comme si elle était heureuse" (p. 169).

Une fois encore, tout finit et tout commence, une chance s'offre, un chaînon est prêt à s'ajouter, comme dans un amour, comme dans une amitié, comme dans un recueil de nouvelles. Et Jean-Pierre Boucher le dit bien: - La mise en présence de textes les éclaire en outrè les uns les autres, leur donne une signification qu'ils n'avaient pas isolément, comme une exposition de tableaux révèle, selon l'accrochage, ce que souvent on n'avait pas encore vu en eux. " 21

20 André Carpentier, op. ctt., p. 36.

21 Jean-Pierre Boucher, op. cit., p. 11. 